\title{
Three Flavor QCD at High Temperatures
}

\author{
The MILC Collaboration: C. Bernard ${ }^{\text {a }}$, T. Burch ${ }^{\mathrm{b}}$, C. DeTar ${ }^{\mathrm{c}}$, Steven Gottlieb ${ }^{\mathrm{d}}$, E.B. Gregory ${ }^{\mathrm{e}}$, \\ U.M. Heller $^{\text {f }}$, J. Osborn ${ }^{\text {c }}$, R.L. Sugar ${ }^{\mathrm{g} *}$, D. Toussaint ${ }^{\mathrm{e}}$ \\ ${ }^{a}$ Department of Physics, Washington University, St. Louis, MO 63130, USA \\ bUniversität Regensburg, Theoretische Physik, 93040 Regensburg, Germany \\ ${ }^{\mathrm{c}}$ Physics Department, University of Utah, Salt Lake City, UT 84112, USA \\ ${ }^{\mathrm{d}}$ Department of Physics, Indiana University, Bloomington, IN 47405, USA \\ eDepartment of Physics, University of Arizona, Tucson, AZ 85721, USA \\ ${ }^{\mathrm{f}}$ American Physical Society, One Research Road, Ridge, NY 11961-9000, USA \\ gDepartment of Physics, University of California, Santa Barbara, CA 93106, USA
}

\begin{abstract}
We have continued our study of the phase diagram of high temperature QCD with three flavors of improved staggered quarks. We are performing simulations with three degenerate quarks with masses less than or equal to the strange quark mass $m_{s}$ and with degenerate up and down quarks with masses $m_{u, d}$ less than the strange quark mass. For the quark masses studied to date, we find a crossover that strengthens as $m_{u, d}$ decreases, rather than a bona fide phase transition. We present new results for the crossover temperature extrapolated to the physical value of $m_{u, d}$, and for quark number susceptibilities.
\end{abstract}

\section{THE PHASE DIAGRAM}

The MILC Collaboration is studying high temperature QCD with three flavors of improved staggered quarks [1] using the Asqtad quark action 2. Simulations are being carried out with lattice spacings $1 / 4 T, 1 / 6 T$ and $1 / 8 T$. We are considering two cases: 1) all three quarks have the same mass $m_{q}$; and 2) the two lightest quarks have equal mass $m_{u, d}$, while the mass of the third quark is fixed at approximately that of the strange quark $m_{s}$. We refer to these cases as $N_{f}=3$ and $N_{f}=2+1$, respectively. We have carried out studies with $0.2 m_{s} \leq m_{q} \leq m_{s}$ for the $N_{f}=3$ case, and with $0.1 m_{s} \leq m_{u, d} \leq m_{s}$ for $N_{f}=2+1$. At the masses we have studied to date, we find rapid crossovers, which sharpen as the quark mass is reduced, rather than a bona fide phase transition. During the past year we have extended and clarified our calculations of

*Presented by R.L. Sugar the quark number susceptibilities, which provide an excellent signal for the crossover, and which are directly related to event by event fluctuations in heavy ion collisions. Comparison of susceptibilities at different lattice spacings show that our results are close to the continuum values. We have also sharpened our estimates of the crossover temperature for both the $N_{f}=3$ and $N_{f}=2+1$ cases.

For both the $N_{f}=3$ and $2+1$ cases we have carried out thermodynamics studies on lattices with four, six and eight times slices, and aspect ratio $N_{s} / N_{t}=2$. Here $N_{s}$ and $N_{t}$ are the spatial and temporal dimensions of the lattice in units of the lattice spacing. At the lightest quark masses, we also performed some simulations with aspect ratio three, and obtained results that are indistinguishable from those with aspect ratio two. For both cases the standard thermodynamic quantities show a crossover from confined behavior at low temperature to deconfined behavior at high 


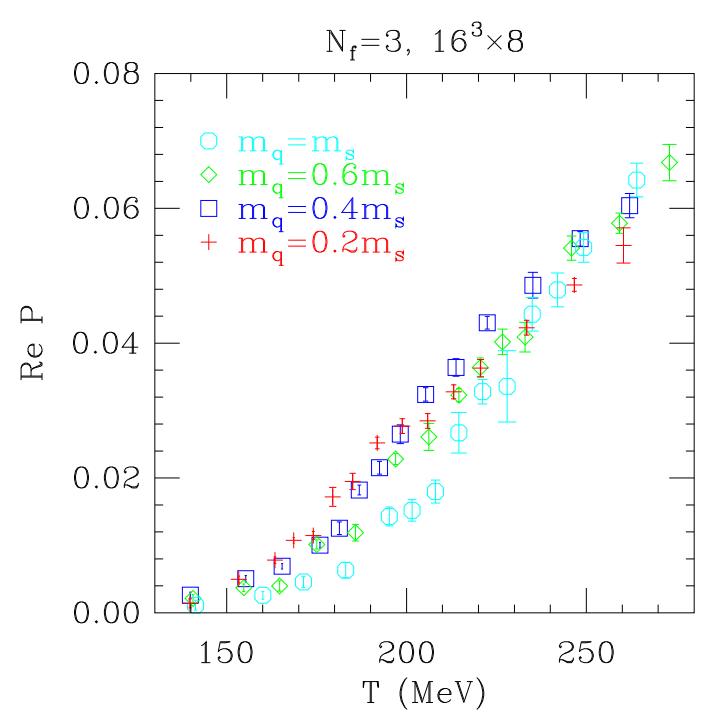

Figure 1. The real part of the Polyakov loop on $16^{3} \times 8$ lattices for $N_{f}=3$.

temperature, as is illustrated in Fig. 11 where we plot the real part of the Polyakov loop for $N_{f}=3$ on $16^{3} \times 8$ lattices. There is a slight trend for the temperature dependence of the Polyakov loop to be steeper for larger quark masses. This is to be expected, since at sufficiently large quark masses, it is a bona fide order parameter. The $\bar{\psi} \psi$ susceptibility $\chi_{\text {tot }}$ provides a clear signal for the crossover. It is given by

$\chi_{\mathrm{tot}}=\frac{\partial}{\partial m}\langle\bar{\psi} \psi\rangle$

We plot this quantity as a function of temperature on $8^{3} \times 4$ lattices in Fig. 2. Note the increase in the height of the peak as the quark mass is decreased. Our work and that of the Bielefeld group [3] strongly suggests that in the real world, $N_{f}=2+1$, there is no phase transition at the physical quark masses. With this assumption, we have estimated the critical temperature for $N_{f}=$ $2+1$ at the physical value of $m_{u, d}$ through an extrapolation of the form

$r_{1} T_{c}=c_{0}+c_{1}\left(m_{\pi} / m_{\rho}\right)^{d}+c_{2}\left(a T_{c}\right)^{2}$,

where we evaluated $T_{c}$ for each value of $N_{t}$ and $m_{\pi} / m_{\rho}$ for which we have made measurements

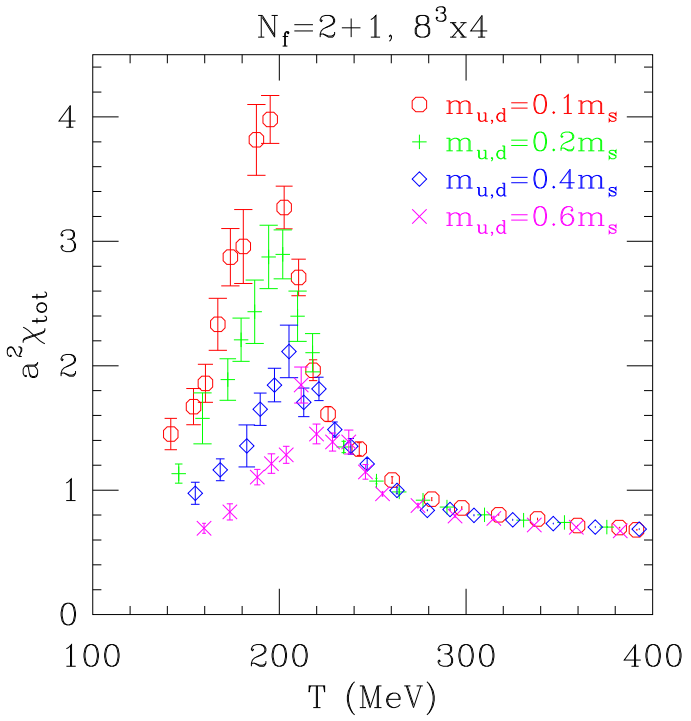

Figure 2. The $\bar{\psi} \psi$ susceptibility as a function of temperature on $8^{3} \times 4$ lattices for $N_{f}=2+1$.

from the peak in the $\bar{\psi} \psi$ susceptibility. For a second order phase transition in the $\mathrm{O}(4)$ universality class at $m_{u, d}=0, d=2 / \beta \delta \approx 1.08$. We find that $T_{c}=169(12)(4) \mathrm{MeV}$ with a $\chi^{2}$ of 2.1 for 11 degrees of freedom. The first error is the fit error, the second from the uncertainty in $r_{1}$, taken as $0.317(7) \mathrm{fm}$ [4. To test the sensitivity of $T_{c}$ to $d$, we have also performed a fit with $d=2$, which yields $T_{c}=174(11)(4) \mathrm{MeV}$ with a $\chi^{2}$ of 1.5 for 11 degrees of freedom. So, the goodness of the fit does not allow us to prefer either of them.

\section{QUARK NUMBER SUSCEPTIBILI- TIES}

In order to study the quark number susceptibilities [56], we introduce chemical potentials $\mu_{\alpha}$ coupled to a set of mutually commuting conserved charges $Q_{\alpha}$. The quark number susceptibilities are related to event-by-event fluctuations in heavy ion collisions 7 by the fluctuationdissipation theorem

$\chi_{\alpha, \beta}(T)=\left\langle\left(Q_{\alpha}-\left\langle Q_{\alpha}\right\rangle\right)\left(Q_{\beta}-\left\langle Q_{\beta}\right\rangle\right)\right\rangle$.

We work at $\mu_{\alpha}=0$, so the brackets, \langle\rangle , in Eq. (3) indicate averages weighted by the standard, real Euclidean action for QCD, and $\left\langle Q_{\alpha}\right\rangle=0$. 
Rather than choosing the three independent charges to be the number operators for up, down and strange quarks, it appears more physical to take them to be the $z$-component of isospin, $Q_{I}$, the hypercharge, $Q_{Y}$, and the baryon number, $Q_{B}$. The rows and columns of the susceptibility matrix, $\chi_{\alpha, \beta}(T)$ are then labeled by $I, Y$ and $B$. In the $N_{f}=3$ case, where $m_{u}=m_{d}=m_{s}$, $\chi$ is a diagonal matrix, and there are no correlations among fluctuations in $Q_{I}, Q_{Y}$ and $Q_{B}$, while for the $N_{f}=2+1$ case, where $m_{u}=m_{d}$, the only correlations are between fluctuations in hypercharge and baryon number. For temperatures below the phase transition or crossover, the lightest particle that can be excited by a chemical potential coupled to $Q_{I}$ is the pion, while for chemical potentials coupled to $Q_{Y}$ and $Q_{B}$ it is the kaon and the nucleon, respectively. Above the transition temperature each of the chemical potentials can excite quark states that are much lighter than hadrons, so we expect the diagonal elements of $\chi$ to increase sharply in the vicinity of the transition, and they do. This is illustrated in Fig. 3 where we plot the diagonal elements of the susceptibility matrix, $\chi_{I, I}, \chi_{Y, Y}$ and $\chi_{B, B}$, as a function of temperature for two light quarks with mass $0.2 m_{s}$ and one heavy quark with mass $m_{s}$ on $12^{3} \times 6$ lattices. $\chi_{Y, Y}$ and $\chi_{B, B}$ have been multiplied by factors of $3 / 4$ and $3 / 2$ respectively, so that the quantities plotted approach the same high temperature limit as $\chi_{I, I}$. Also shown is $\chi_{Y, B}$, the only non-zero off-diagonal matrix element of $\chi$ for $m_{u}=m_{d}$. It measures correlations between fluctuations in the hypercharge and baryon number. The coefficient of $\chi_{Y, B}$ in this figure is the geometric mean of those for $\chi_{Y, Y}$ and $\chi_{B, B}$. The good agreement between these results, and similar ones on lattices with eight time slices, indicate that they are close to their physical values.

This work is supported by the US National Science Foundation and Department of Energy and used computer resources at Florida State University (SP), Indiana University, NCSA, NERSC, NPACI (Michigan), FNAL, and the University of Utah (CHPC).

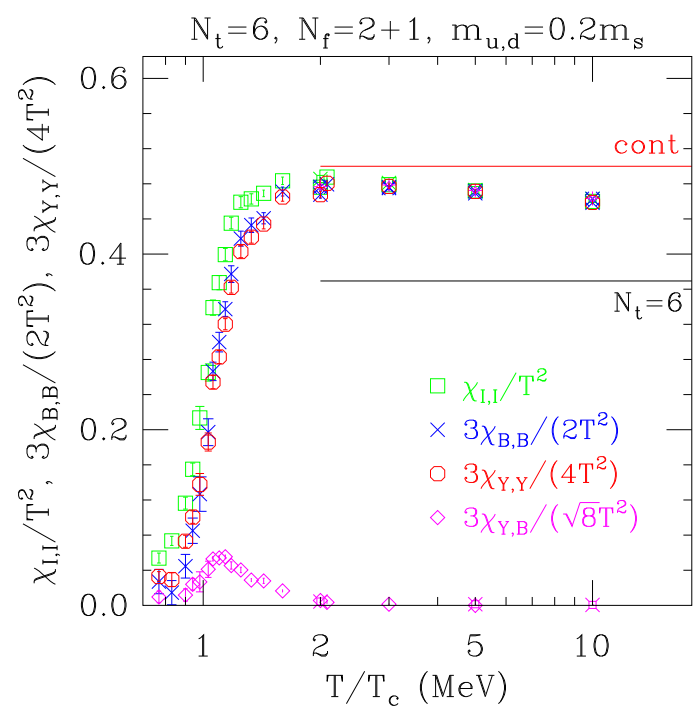

Figure 3. The non-vanishing elements of the susceptibility matrix for two light quarks of mass $0.2 m_{s}$ and one heavy quark of mass $m_{s}$ on $12^{3} \times 6$ lattices.

\section{REFERENCES}

1. Earlier reports on this work can be found in The MILC Collaboration, C. Bernard, et al., Nucl. Phys. B (Proc. Suppl.) 106 (2002) 429; 119 (2003) 613; 129 \& 130 (2004) 626; and hep-lat/0405029.

2. G.P. Lepage, Phys. Rev. D 59 (1999) 074501; Kostas Orginos, Doug Toussaint and R.L. Sugar, Phys. Rev. D 60 (1999) 054503.

3. F. Karsch et al., Nucl. Phys. B (Proc. Suppl.) 129\& 130 (2004) 614.

4. The MILC Collaboration: C. Bernard, et al., hep-lat/0402030 to be published in Phys. Rev. D.

5. S. Gottlieb et al., Phys. Rev. Lett. 59 (1987) 2247.

6. R.V. Gavai and S. Gupta, Phys. Rev. D 64 (2001) 074506; Phys. Rev. D 65 (2002) 094515; R.V. Gavai, S. Gupta and P. Majumdar, Phys. Rev. D 65 (2002) 054506.

7. B. Müller, Nucl. Phys. A 702 (2002) 281; V. Koch, M. Bleicher and S. Jeon, Nucl. Phys. A 702 (2002) 291; R.V. Gavai, Nucl. Phys. A 702 (2002) 299. 